\title{
Mobility of Ferroelectric Domains in Antimony Sulfoiodide
}

\author{
B. Toroń, M. Nowak, M. Kępińska AND P. SzPERlich \\ Solid State Physics Section, Institute of Physics - Center for Science and Education, Silesian University of \\ Technology, Z. Krasińskiego 8, 40-019 Katowice, Poland
}

\begin{abstract}
Different optical energy gaps in ferroelectric and paraelectric phases as well as light scattering on domain walls allow to observe ferroelectric domains in antimony sulfoiodide (SbSI) near the Curie temperature. Mobility $8.11(44) \times 10^{-8} \mathrm{~m}^{2} /(\mathrm{V} \mathrm{s})$ of ferroelectric domain walls under external electric field has been determined along $c$-axis of SbSI single crystals using optical transmittance microscopy.
\end{abstract}

DOI: 10.12693/APhysPolA.126.1093

PACS: 77.80.-e, 77.80.Dj, 77.80.B-, 81.05.Hd

\section{Introduction}

Nowadays, there is a growing interest in application of ferroelectric semiconductors in electronics, e.g. in diodes, photodiodes, and ferroelectric random access memories $[1,2]$. These applications are determined e.g. by domain mobilities. Antimony sulfoiodide (SbSI) is a promising semiconducting [3] ferroelectric [4] with many interesting properties, e.g. piroelectric, pyrooptic, electrooptic, photoferroelectric effects, and gas sensing $[5,6]$.

Different optical energy gaps in ferroelectric and paraelectric phases as well as light scattering on the domain walls allow to observe ferroelectric domains in ferroelectric materials e.g. in SbSI $[7,8]$. In this paper the mobility of ferroelectric domains in SbSI has been determined for the first time using optical transmittance near the Curie temperature $\left(T_{\mathrm{C}}=293 \mathrm{~K}[4]\right)$.

\section{Experimental details}

The investigated SbSI single crystals have been grown from vapour phase. Optical transmittance of SbSI single crystals have been measured using PC2000 Ocean Optics Inc. spectrophotometer equipped with master card with 600 lines grating. The spectrophotometer has been equipped with appropriate waveguide cables and deuterium-halogen light source DH2000-FHS from Ocean Optics Inc. The experimental setup has been equipped with Oriel Glan-Thompson polarizer to perform the measurements for linearly polarized radiation with electric field parallel and perpendicular to the $c$-axis of the crystals. The sample has been mounted in $10^{-2} \mathrm{~Pa}$ vacuum obtained by turbomolecular pump TSH 071E (Pfeifffer Vacuum) in optical D2209 chamber of R2205 Cryogenic Microminiature Refrigeration II-B System based on Joule Thomson effect (MMR Technologies, Inc.). K77 controller (MMR Technologies, Inc.) has been used to monitor the temperature during optical investigations.

Electrodes made of High Purity Silver Paste 05002AB from SPI Supplies have been deposited on SbSI single crystal planes perpendicular to c-axis of the crystal for investigations of the ferroelectric domain mobility. In these investigations SbSI sample has been mounted on the Peltier element cooled with K20 ThermoScientific ultrathermostat. Sample temperature has been measured by Pt100 sensor connected to Peltron GmbH 100/10 RS Peltier driver. Keithley 2410C SourceMeter has been used as the bias source. Domain motion has been observed and recorded in transmitted linearly polarized VIS light by Carl Zeiss Stemi 2000C microscope equipped with Olympus DP25 digital camera. Measurements have been performed below and above the Curie temperature of SbSI single crystal for plane polarized light with electric field parallel and perpendicular to $c$-axis of the crystal.

\section{Results and discussion}

Figure 1 presents the decrease of optical transmittance near the Curie point of SbSI single crystal illuminated with plane polarized light with electric field parallel to the $c$-axis. Qualitatively similar but less visible effect has been observed for perpendicular polarization of light due to weaker interaction of such polarized light with highly anisotropic SbSI crystal lattice. Nature of the decrease of optical transmittance near the Curie point of SbSI is explained by light scattering on ferroelectric domain walls. One can observe such walls in the temperature range of $2 \mathrm{~K}$ near to the Curie point (Fig. 2). At temperatures lower than $292 \mathrm{~K} \mathrm{SbSI}$ single crystal is ferroelectric while at temperatures higher than $294 \mathrm{~K}$ is paraelectric. In temperatures between 292 and $294 \mathrm{~K}$ both phases coexist in SbSI single crystal. Domain walls are visible for plane polarized light with electric field parallel and perpendicular to the $c$-axis of SbSI single crystal as well as for nonpolarized light, whereas the best visibility is achieved for plane polarized light with electric field parallel to the $c$-axis of SbSI crystal. Angles between domain walls and $c$-axis of SbSI crystal are observed mainly for $67^{\circ}$ and $90^{\circ}$. Such values correspond to the crystallographic planes (101) and (001) of SbSI single crystal, respectively [9].

External electric field with large enough intensity applied along $c$-axis of SbSI crystal at the Curie temperature leads to domain movement (Fig. 3). No domain motion has been observed for electric fields smaller than 


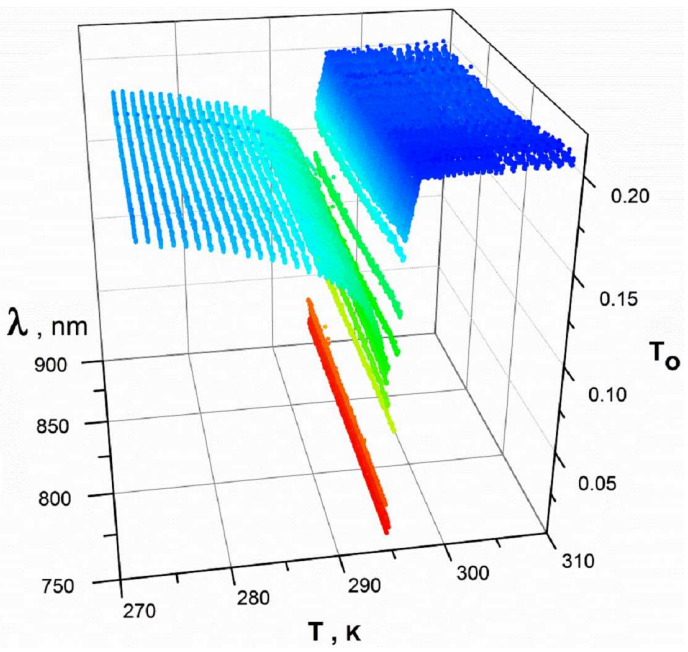

Fig. 1. Spectral and temperature dependences of optical transmittance of SbSI single crystal illuminated with plane polarized light with electric field parallel to the $c$-axis.

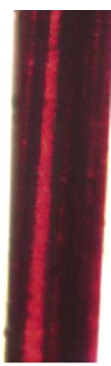

$291.5 \mathrm{~K}$

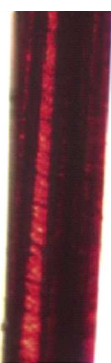

$292.0 \mathrm{~K}$

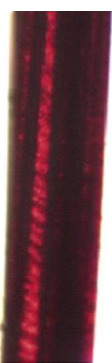

$292.5 \mathrm{~K}$

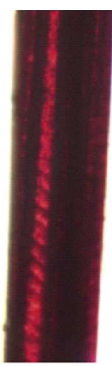

$293.0 \mathrm{~K}$

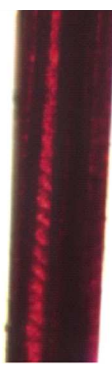

$293.5 \mathrm{~K}$

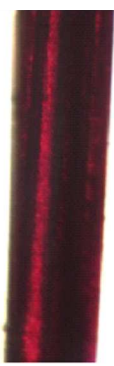

$294.0 \mathrm{~K}$
Fig. 2. Images of domain walls in SbSI single crystal recorded at different temperatures in transmitted plane polarized light with electric field parallel to the $c$-axis without applying external electric field.

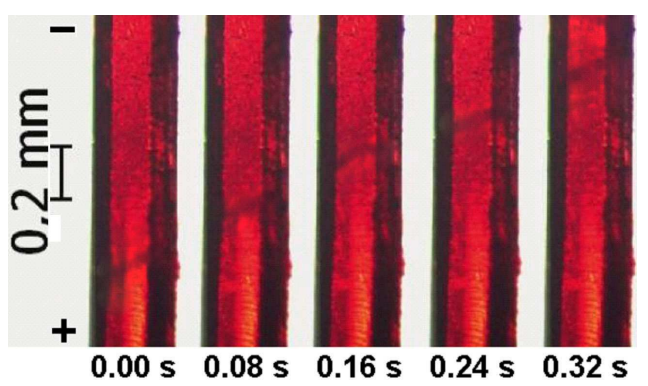

Fig. 3. Positions of a single ferroelectric domain wall recorded subsequently in SbSI single crystal for external electric field $E=50 \mathrm{kV} / \mathrm{m}$ applied along the $c$-axis (the snapshots were done for transmitted plane polarized light with electric field parallel to the $c$-axis of SbSI single crystal; $T=293 \mathrm{~K}$; symbols + , - present the bias polarity).

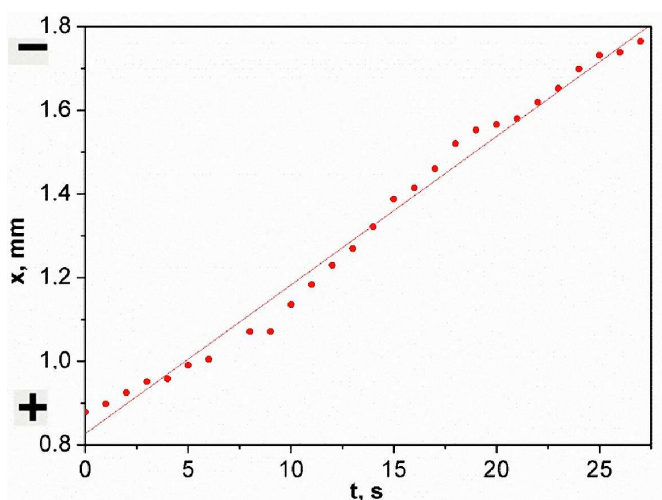

Fig. 4. Position of domain wall in SbSI single crystal vs. time (electric field $E=15 \mathrm{kV} / \mathrm{m}$ applied along the $c$-axis; $T=293 \mathrm{~K}$; Solid line represents the fitted linear dependence; $v=\Delta x / \Delta t=3.554(78) \times 10^{-2} \mathrm{~mm} / \mathrm{s}$; symbols,+- present the bias polarity).

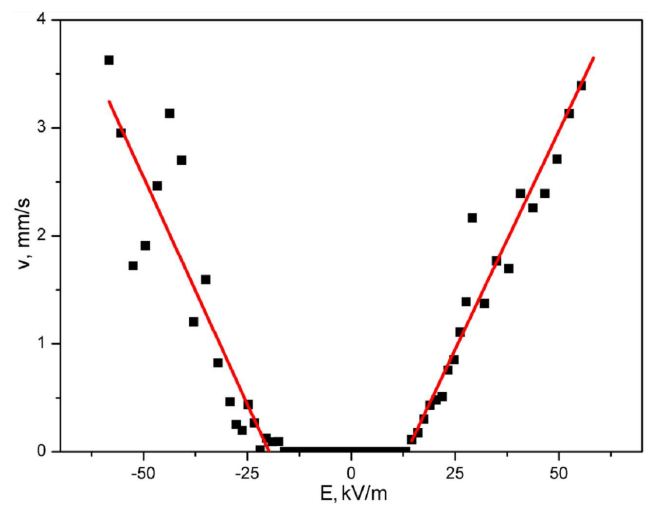

Fig. 5. Domain walls velocity in SbSI single crystal vs. external electric field applied along the $c$-axis (solid lines represent fitted theoretical dependence (1); values of fitted parameters in the text; $T=293 \mathrm{~K}$ ).

coercive field $E_{\mathrm{C}}=13 \mathrm{kV} / \mathrm{m}$. Such field intensity corresponds to energy required to move domain border fixed on crystal structure defects. For electric field higher than $E_{\mathrm{C}}$, domain walls move toward positive or negative electrode depending on electric charge accumulated on them. The linear dependence of the position of domain wall in SbSI single crystal on time (Fig. 4) allows to determine velocity of the domain motion. Figure 5 presents domain walls velocity $v(E)$ as a function of the applied external electric field intensity $(E)$. Domain walls mobility $(\mu)$ can be determined from this characteristic by least square fitting of appropriate linear dependences with the following formula [10]:

$$
v(E)=\mu E+b .
$$

Solid lines in Fig. 5 represent the fitting obtained for $\mu=8.11(44) \times 10^{-8} \mathrm{~m}^{2} /(\mathrm{Vs})$.

It should be noted that some of the domain walls are fixed on defects of crystal structure. The creation of two domain walls and a new area between them may 
also occur in any place of SbSI single crystal. Merging and splitting of ferroelectric domains have also been observed in $\mathrm{LaTiO}_{3}$ [11]. In strong external electric field $\left(E>2 E_{\mathrm{C}}\right)$ domain walls move to crystal edge and the whole crystal becomes a single domain - the ferroelectric sample is formed. Domain walls return to their initial position when external electric field is switched off.

\section{Conclusions}

For the first time mobility of ferroelectric domains has been determined in SbSI single crystal. The fitted mobility $\mu=8.11(44) \times 10^{-8} \mathrm{~m}^{2} /(\mathrm{Vs})$ of ferroelectric domains in SbSI single crystal is four orders of magnitude smaller than the observed in $\mathrm{BaTiO}_{3}[10]$ and two orders of magnitude smaller than that in $\mathrm{LaTiO}_{3}$ [11].

It should be underlined that due to the light scattering on domain walls, one should very carefully interpret the temperature dependences of optical parameters (e.g. absorption coefficient and refractive index) determined from optical transmittance or reflectance data near the Curie point.

\section{Acknowledgments}

This work was partially supported by NCN grant No. DEC-2011/01/B/ST5/06273.

\section{References}

[1] J.F. Scott, Science 315, 954 (2007).

[2] B. Toroń, M. Nowak, A. Grabowski, M. Kępińska, Acta Phys. Pol. A 124, 830 (2013).

[3] M. Nowak, P. Szperlich, Opt. Mater. 35, 1200 (2013).

[4] P. Szperlich, M. Nowak, Ł. Bober, J. Szala, D. Stróż, Ultrason. Sonochem. 16, 398 (2009).

[5] M. Nowak, K. Mistewicz, A. Nowrot, P. Szperlich, M. Jesionek, A. Starczewska, Sensor Actuat. A-Phys. 210, 32 (2014).

[6] M. Nowak, A. Nowrot, P. Szperlich, M. Jesionek, M. Kępińska, A. Starczewska, K. Mistewicz, D. Stróż, J. Szala, T. Rzychoń, E. Talik, R. Wrzalik, Sensor Actuat. A-Phys. 210, 119 (2014).

[7] K. Imai, S. Kawada, M. Ida, J. Phys. Soc. Jpn. 21, 1855 (1966).

[8] M. Nowak, P. Szperlich, A. Kidawa, M. Kępińska, P. Gorczycki, B. Kauch, Proc. SPIE 5136, 172 (2003).

[9] E.I. Gerzanich, V.A. Lyakhovitskaya, V.M. Fridkin, B.A. Popovkin, in: Current Topics in Materials Science, Vol. 10, Ed. E. Kaldis, North-Holland, Amsterdam 1982, p. 55 .

[10] L. Bakaleinikova, A. Gordon, Physica B 388, 359 (2007).

[11] V. Gopalan, T.E. Mitchell, J. Appl. Phys. 85, 2304 (1999). 